\title{
Variable frequency stimulation of sacral neuromodulation in black-zone overactive bladder patients: a case report
}

\author{
Lingfeng Meng, Tongxiang Diao, Miao Wang, Xiaodong Liu, Wei Zhang, Zijian Tian, Jianye Wang, \\ Yaoguang Zhang \\ Department of Urology, Beijing Hospital, National Center of Gerontology, Institute of Geriatric Medicine, Chinese Academy of Medical Sciences, \\ Beijing, China \\ Correspondence to: Yaoguang Zhang. Department of Urology, Beijing Hospital, National Center of Gerontology, Institute of Geriatric Medicine, \\ Chinese Academy of Medical Sciences, No. 1 Dongdan Dahua Road, Dongcheng District, Beijing 100730, China. Email: zhang003887@sina.com.
}

\begin{abstract}
Overactive bladder (OAB) is a common urological disease, reducing patient quality of life (QoL). Sacral neuromodulation (SNM) is a treatment option used when conservative treatment is inadequate. However, constant frequency stimulation-SNM (CFS-SNM) may not be sufficiently effective in achieving targeted symptom reduction in some patients. For such black-zone patients, a different treatment strategy is needed. Variable frequency stimulation (VFS) has been used for deep-brain stimulation treatment in patients with Parkinson's disease with positive outcomes. Accordingly, in this study, we hypothesized the promising outcomes of VFS-SNM in black-zone OAB patients. Here, we evaluated the efficacy and safety of VFS-SNM viz-a-viz CFS-SNM in a black-zone patient with refractory OAB whose frequent micturition symptoms were not relieved after undergoing traditional conservative treatment. A 50-year-old male patient was treated with CFS-SNM at our hospital in October 2016, but his symptoms recurred after administering multiple medications and program-controlled parameter adjustments. We then treated the patient with VFSSNM in March 2020. A 2-week follow-up through telephonic interviews was conducted; the improvements in voiding symptoms were evaluated by calculating the OAB symptom score (OABSS) and OAB-related QoL (OAB-QoL) score. We observed that OABSS was significantly lower after VFS-SNM than after CFS-SNM. Further, we observed that VFS-SNM significantly improved daytime and nocturnal micturition frequency, as evident from the reduced OABSSs from after CFS-SNM to after VFS-SNM. The main reason for patient dissatisfaction after CFS-SNM was the increased average daily micturition frequency and urgency. VFSSNM controlled the micturition frequency to within the patient's acceptable range, significantly improving the patient's QoL (40\% improvement in OAB-QoL score). To the best of our knowledge, this is the first case report on the use of VFS-SNM with positive outcomes in a black-zone OAB patient, suggesting that VFSSNM is not inferior to CFS-SNM in the treatment of black-zone patients.
\end{abstract}

Keywords: Constant frequency stimulation (CFS); overactive bladder (OAB); sacral neuromodulation (SNM); variable frequency stimulation (VFS); case report

Submitted Jun 08, 2020. Accepted for publication Oct 06, 2020.

doi: $10.21037 /$ tau-20-889

View this article at: http://dx.doi.org/10.21037/tau-20-889

\section{Introduction}

Overactive bladder $(\mathrm{OAB})$ is a common disease in urology and is defined by the International Continence Society as urinary urgency usually with urination frequency, with or without urgent urinary incontinence and without any urinary tract infection or other obvious pathological changes (1). The syndrome has become an increasingly serious problem worldwide. It is estimated that the prevalence rate of $\mathrm{OAB}$ among American adults is approximately $16.9 \%$ and increases with age (2). In China, the prevalence of $\mathrm{OAB}$ in adults is between $4.7 \%$ and $6.0 \%$; 
Table 1 Scores in different periods

\begin{tabular}{lccc}
\hline Score & Baseline & Constant frequency & Variable frequency \\
\hline OABSS & & & 1 \\
Q1 (daytime frequency) & 2 & 3 & 2 \\
Q2 (nocturnal frequency) & 3 & 2 & 2 \\
Q3 (urgency) & 3 & 1 & 1 \\
Q4 (urgency incontinence) & 2 & 5 & 3 \\
OAB-QoL & 8 & 5
\end{tabular}

OABSS, overactive bladder symptom score; OAB-QoL, overactive bladder-related quality of life score.

among these adults, $19.9 \%$ are over the age of 40 years (3). The disease negatively affects patients' quality of life (QoL) and increases the socio-economic health burden.

Electrical stimulation therapy has been used for more than 50 years since its first application by Caldwell for the treatment of urinary symptoms in 1963 (4). Current studies have shown that sacral neuromodulation (SNM) is a safer and more effective treatment for $\mathrm{OAB}$ in patients who are unresponsive to traditional conservative treatments $(5,6)$. However, there is still a considerable proportion of patients who have previously undergone SNM treatment and still experience either recurrent symptoms or weakening of the degree of symptom improvement over time $(7,8)$. Existing treatments for these patients include combining oral drugs; adjusting the stimulation frequency, intensity, and time; and other program-controlled methods. However, there are some patients (defined as black-zone patients) in whom none of the above treatment options are sufficiently effective in achieving targeted symptom reduction.

The successful development of a new type of SNM system (PINS, Beijing, China) with variable frequency stimulation (VFS) function has provided a new direction for the diagnosis and treatment of black-zone $\mathrm{OAB}$ patients. In March 2020, our hospital provided VFS-SNM to a blackzone OAB patient, and the ensuing follow-up outcomes were promising. In this report, we discuss the efficacy of using VFS-SNM over constant frequency stimulation (CFS)-SNM in the treatment of a black-zone OAB patient. We present the following case in accordance with the CARE reporting checklist (available at http://dx.doi.org/10.21037/ tau-20-889).

\section{Case presentation}

A 50-year-old male patient presented to our hospital with frequent urination and nocturia for more than 10 years and progressive aggravation of symptoms for 3 years. Oral drugs, behavioral therapy, and other conservative treatments had been ineffective. In October 2016, after obtaining informed consent from the patient, SNM (PINS, G131) was performed. Two years later, the patient's symptoms had worsened. Upon treatment with CFS-SNM, the patient was still urinating up to 11 times in day and 3 times at night; the patient remained dissatisfied even after multiple medication and program-controlled parameter adjustments. Hence, we upgraded the patient to the VFS-SNM program in March 2020 and followed-up on his progress through the telephone for 2 weeks after the treatment. Our results show a significant improvement in the symptoms and scores from those obtained after the CFS-SNM treatment. Furthermore, there were no operation-related complications.

We evaluated the OABSS (9) and OAB-QoL scores (10) of the patient at three stages [before SNM (baseline), after CFS-SNM, and after VFS-SNM]. An interference question on the OAB-QoL, "Overall, how much do your urinary symptoms interfere with your everyday life?", was measured on a scale from 0 to 10 . The two stimulation modes used in this case study were negative stimulation modes. The scores in all three stages and the detailed parameters of different stimulation modes are shown in Tables 1,2.

Both stimulation methods were found to significantly reduce the OABSS; compared with the baseline, the improvement rate after CFS-SNM was $30 \%$ and that after VFS-SNM was 50\%. Further, VFS-SNM significantly improved both the daytime and nocturnal urination frequency. The score for Question 1 (daytime frequency) decreased from 2 points at baseline to 0 points after VFS-SNM, whereas the score for Question 2 (nocturnal frequency) decreased from 3 to 2 points. In contrast, the 
Table 2 Detailed parameters for different treatment modes

\begin{tabular}{lcc}
\hline Parameters & Constant frequency & Variable frequency \\
\hline Stimulation contact & 1,3 & 1,3 \\
Current $(\mathrm{mA})$ & 0.5 & 0.3 \\
Frequency $(\mathrm{Hz})$ & 8 & $8,14,30$ \\
Pulse width $(\mu \mathrm{s})$ & 350 & 350 \\
\hline
\end{tabular}

The variable frequency stimulation applied was $8 \mathrm{~Hz}$ for $20 \mathrm{~s}, 14 \mathrm{~Hz}$ for $10 \mathrm{~s}$, and $30 \mathrm{~Hz}$ for $5 \mathrm{~s}$ per cycle.

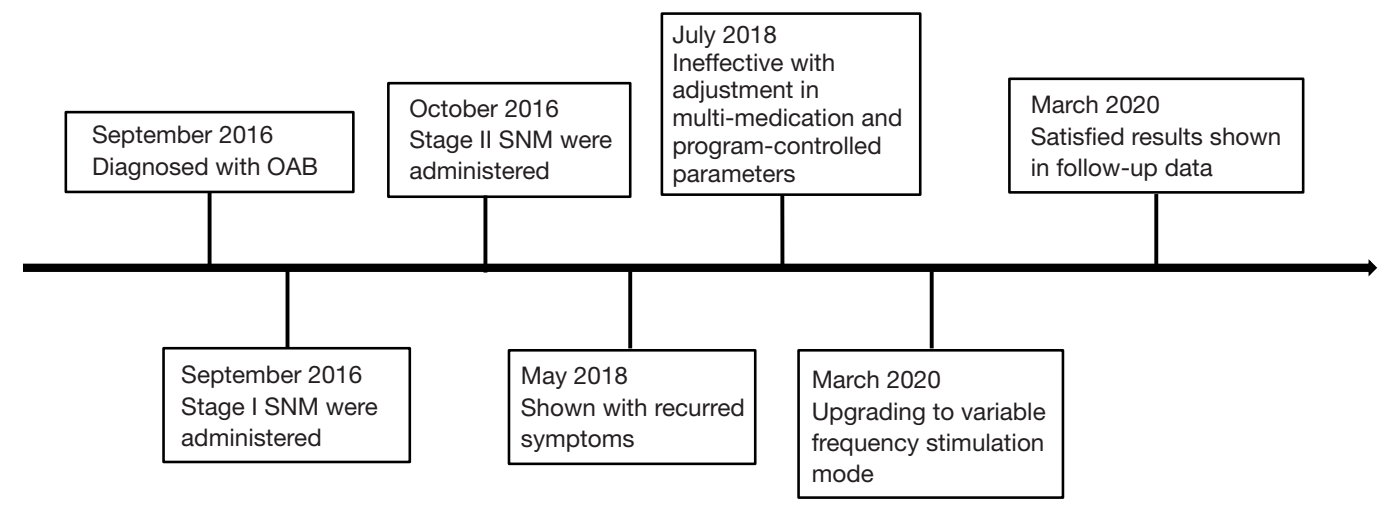

Figure 1 The timeline picture of this case. OAB, overactive bladder; SNM, sacral neuromodulation.

CFS-SNM treatment was less effective in improving these symptoms (the Q1 score decreased from 2 points to 1 point, and the Q2 score remained at 3 points). Moreover, on comparing the OAB-QoL scores, we found that VFSSNM significantly reduced the patient's OAB-QoL score. The improvement rate of OAB-QoL score after VFS-SNM treatment was $62.5 \%$, while that after CFS-SNM was only $37.5 \%$. The timeline picture was shown in Figure 1.

All procedures performed in studies involving human participants were in accordance with the ethical standards of the institutional and/or national research committee(s) and with the Helsinki Declaration (as revised in 2013). Written informed consent was obtained from the patient.

\section{Discussion}

SNM has been used in the treatment of OAB for more than 10 years. With the rapid development of SNM therapy, it has become more popular in China in the past 4 years. However, there is still a considerable proportion of patients who receive SNM but cannot achieve satisfactory clinical efficacy after repeated treatments. We define these as blackzone patients. Effective treatment of these patients remains problematic for urologists.

Jia et al. of Tsinghua University first proposed VFS in 2015. In their study, patients with Parkinson's disease received subthalamic nucleus-deep-brain stimulation after which the postoperative symptoms were analyzed. Even after repeatedly adjusting the stimulation parameters, when the improvement of clinical symptoms was not obvious, they attempted to use VFS at $60 \mathrm{~Hz}$ for $20 \mathrm{~s}$ and at $130 \mathrm{~Hz}$ for $30 \mathrm{~s}$ per cycle. Their results showed that VFS can significantly improve the clinical symptoms in patients more effectively than the traditional CFS (11). Similar conclusions were subsequently drawn by Wang et al. (12). In a more recent study, Jia et al. performed a randomized controlled trial to compare variable and constant frequency deep-brain stimulation for treating patients with advanced Parkinson's disease (13). Based on these studies, we hypothesized that VFS could be applied to the field of urology, particularly in the case of black-zone patients, and may enable clinicians to obtain better postoperative results.

In our case, the patient's symptoms improved more satisfactorily because the VFS-SNM controlled the frequency of urination within the range deemed acceptable by the patient, which greatly improved the QoL. After a 


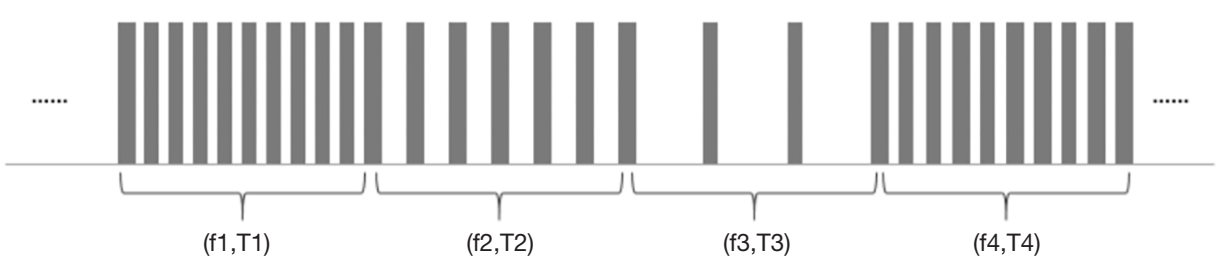

Figure 2 Each set of variable frequency simulation includes two parameters: stimulation frequency (fn) and stimulation time (Tn).

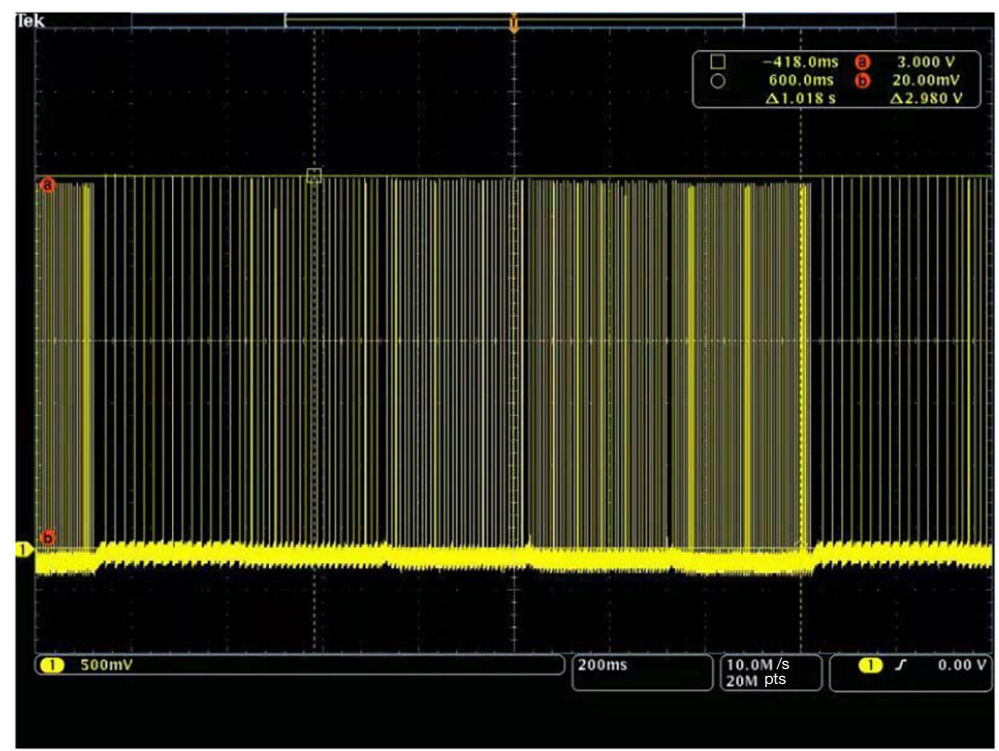

Figure 3 Typical pulse output waveform.

thorough literature search, we found that ours was likely the first study to apply VFS-SNM for the treatment of urological diseases. Therefore, to improve the use of VFS in the clinical environment, we have explained the technology of the VFS-SNM system below.

VFS does not strictly refer to the alternation of highand low-frequency bands; it can also be the alternation of relatively high and low frequency. The duration of frequency band stimulation is also one of the parameters that need to be adjusted. The main technical parameters of VFS are set as follows: frequency range, $2-250 \mathrm{~Hz}$ (62 levels adjustable); and duration of each frequency point, $0.1 \mathrm{~s}-24 \mathrm{~h}$ (145 levels adjustable). In the same program group, alternate stimulation can be performed at 1 to 5 different frequency points. The new SNM system with VFS function (PINS, Beijing, China) diversifies the selection of the programmed parameters and is expected to further improve the curative effect.
The VFS function is completely software controlled, and the hardware is no different from the traditional stimulation pulse generator hardware. The software realizes the function of VFS by adding a new timing task to switch the stimulus frequency at different times. At present, the VFS-SNM system can set 2-5 groups of different variable frequency parameters. Each group of variable frequency parameters includes two parameters: stimulation frequency (fn) and stimulation time (Tn) (Figure 2). Each set of variable frequency parameters (fn, Tn) is independent of each other. The typical pulse output waveform is shown in Figure 3.

Fasano et al. (14) indicated that frequency modulation is the encoding of information in a carrier wave by varying the instantaneous frequency of the wave. In our study, the beneficial results lasted for 2 weeks. Based on our observations of this patient, we believe that for patients who have been treated with relatively low-frequency stimulation 
for a long time, two different frequencies of stimulation can be carried out in this low-frequency band when performing the VFS-SNM. The frequencies are selected based on the patient's subjective sense of stimulation. After the patient adapts to the low-frequency band, the frequency is gradually increased to a relatively higher band to avoid sudden fluctuations in the patient's symptoms as a result of the sudden increase in stimulation parameters. We expect a gradual adaptation of the patients to this new treatment model.

This study has some limitations. First, as this was a case report, the findings cannot be generalized. Second, the follow-up time was quite short; therefore, the longterm effectiveness and safety of VFS-SNM could not be determined. Last, our study failed to clarify the potential mechanism of VFS-SNM in improving symptoms in the black-zone OAB patients. Further randomized controlled trials with large samples are needed to provide a more convincing evidence base for the treatment of these patients.

\section{Conclusions}

We report the first known use of VFS-SNM technology for the treatment of a urological disease with satisfactory results. VFS-SNM was not inferior to CFS-SNM in the treatment of black-zone $\mathrm{OAB}$ patients. Our research team is currently conducting a randomized controlled study on the effectiveness and safety of VFS-SNM that aims at verifying the efficacy of VFS-SNM and improving the operating specifications, while providing new treatment ideas and methods for urological diseases.

\section{Acknowledgments}

Funding: This work was supported by National Key R\&D Program of China (Grant Numbers: 2018YFC2002202, 2016YFC0105505).

\section{Footnote}

Reporting Checklist: The authors have completed the CARE reporting checklist. Available at http://dx.doi.org/10.21037/ tau-20-889

Conflicts of Interest: All authors have completed the ICMJE uniform disclosure form (available at http://dx.doi. org/10.21037/tau-20-889). The authors have no conflicts of interest to declare.
Ethical Statement: The authors are accountable for all aspects of the work in ensuring that questions related to the accuracy or integrity of any part of the work are appropriately investigated and resolved. All procedures performed in studies involving human participants were in accordance with the ethical standards of the institutional and/or national research committee(s) and with the Helsinki Declaration (as revised in 2013). Written informed consent was obtained from the patient.

Open Access Statement: This is an Open Access article distributed in accordance with the Creative Commons Attribution-NonCommercial-NoDerivs 4.0 International License (CC BY-NC-ND 4.0), which permits the noncommercial replication and distribution of the article with the strict proviso that no changes or edits are made and the original work is properly cited (including links to both the formal publication through the relevant DOI and the license). See: https://creativecommons.org/licenses/by-nc-nd/4.0/.

\section{References}

1. Lee UJ, Scott VC, Rashid R, et al. Defining and managing overactive bladder: disagreement among the experts. Urology 2013;81:257-62.

2. Stewart WF, Van Rooyen JB, Cundiff GW, et al. Prevalence and burden of overactive bladder in the United States. World J Urol 2003;20:327-36.

3. Wang $\mathrm{Y}, \mathrm{Xu} \mathrm{K}, \mathrm{Hu} \mathrm{H}$, et al. Prevalence, risk factors, and impact on health related quality of life of overactive bladder in China. Neurourol Urodyn 2011;30:1448-55.

4. CALDWELL KP. The electrical control of sphincter incompetence. Lancet 1963;2:174-5.

5. Haylen BT, de Ridder D, Freeman RM, et al. An International Urogynecological Association (IUGA)/ International Continence Society (ICS) joint report on the terminology for female pelvic floor dysfunction. Int Urogynecol J 2010;21:5-26.

6. Gormley EA, Lightner DJ, Faraday M, et al. Diagnosis and treatment of overactive bladder (non-neurogenic) in adults: AUA/SUFU guideline amendment. J Urol 2015;193:1572-80.

7. Zhang Y, Zhang P, Tian X, et al. Remotely programmed sacral neuromodulation for the treatment of patients with refractory overactive bladder: a prospective randomized controlled trial evaluating the safety and efficacy of a novel sacral neuromodulation device. World J Urol 2019;37:2481-92. 
8. Meng L, Zhang W, Zhang Y, et al. Analysis of the Correlation Between the Clinical Effect of Sacral Neuromodulation and Patient Age: A Retrospective Multicenter Study in China. Neuromodulation 2020. [Epub ahead of print]. doi: 10.1111/ner.13130

9. Homma Y, Yoshida M, Seki N, et al. Symptom assessment tool for overactive bladder syndrome--overactive bladder symptom score. Urology 2006;68:318-23.

10. Coyne K, Revicki D, Hunt T, et al. Psychometric validation of an overactive bladder symptom and healthrelated quality of life questionnaire: the OAB-q. Qual Life Res 2002;11:563-74.

11. Jia F, Guo Y, Wan S, et al. Variable frequency stimulation

Cite this article as: Meng L, Diao T, Wang M, Liu X, Zhang W, Tian Z, Wang J, Zhang Y. Variable frequency stimulation of sacral neuromodulation in black-zone overactive bladder patients: a case report. Transl Androl Urol 2020;9(6):2842-2847. doi: 10.21037/tau-20-889 of subthalamic nucleus for freezing of gait in Parkinson's disease. Parkinsonism Relat Disord 2015;21:1471-2.

12. Wang H, Jiang $Y$, Zhang X, et al. The effect of variable frequency stimulation of subthalamic nucleus on freezing gait of patients with Parkinson's disease. Chin J Neurosurg 2017;33:1124-7.

13. Jia F, Zhang J, Wang H, et al. Variable- versus constantfrequency deep-brain stimulation in patients with advanced Parkinson's disease: study protocol for a randomized controlled trial. Trials 2019;20:749.

14. Fasano A, Lozano AM. The FM/AM world is shaping the future of deep brain stimulation. Mov Disord 2014;29:161-3. 